\title{
EVALUACIÓN DE LA INFORMACIÓN CONTENIDA EN SEIS SEDES WEB DE LAS ESCUELAS UNIVERSITARIAS Y FACULTADES DE BIBLIOTECONOMÍA Y DOCUMENTACIÓN ESPAÑOLAS
}

\author{
Lourdes Castlllo Blasco*, M. J. Martínez de Pablos*", Gloria Server \\ Resumen: Se evalúa la información contenida en las sedes web de seis Escue- \\ las Universitarias y Facultades de Documentación españolas. Se destaca la \\ dificultad que entraña cualquier análisis de este tipo debido a la continua ac- \\ tualización de estos recursos, que dejan la sede temporalmente fuera de ser- \\ vicio.
}

Palabras clave: sedes web, biblioteconomía, documentación.

\begin{abstract}
An evaluation of the information of the web sites of six Spanish University Schools and Faculties of Documentation is presented. The difficulty of an analysis of this type is principally due to the continuous updating of the resources which means that the site is temporarily out of service.
\end{abstract}

Key words: web sites, library science, documentation.

\section{Introducción}

Documentalistas y bibliotecarios, dedicados por su profesión a organizar la información y a informar, son los profesionales mejor preparados para la recuperación y la distribución de información en Internet. La gran heterogeneidad y la abundancia de recursos informativos suponen algunos de los problemas principales que dificultan el acceso a información fiable y útil, que los sistemas de recuperación de información distribuida actuales no permiten diferenciar de la información irrelevante de Internet.

Uno de los primeros dispositivos que facilitan la detección y recuperación de recursos informativos distribuidos en la Red son las listas de recursos. Los grupos profesionales y las instituciones docentes suelen mantener listas de sedes web, a veces dotadas de mecanismos de recuperación sofisticados, a veces sólo meras relaciones alfabéticas. Sin embargo, el carácter especializado de estos dispositivos los dotan de cierto valor para los profesionales o estudiantes que desean un acceso organizado a los recursos accesibles en Internet.

Estas páginas, como las mantenidas por organismos e instituciones académicas y de investigación en España, son el resultado de un proceso de búsqueda, selección, descripción y mantenimiento, que supera la abundancia de recursos, la falta de criterios de validez de estos mismos recursos y la movilidad del medio Internet, con continuas apariciones, traslados y ceses de sedes.

El presente trabajo se propone una evaluación de las sedes web de las institu-

\footnotetext{
* Castillo@rtvv.es.

** Unidad de documentación. RTVV. Polígono de acceso a Ademuz, s/n. 46100 Burjassot.

Recibido: 15-3-99.
} 
ciones españolas correspondientes a centros de formación en Biblioteconomía y Documentación, en función de los recursos que cada una recopila y ofrece a sus usuarios.

\section{Método}

Se entiende por sede web el conjunto de páginas ligadas jerárquicamente, que constituyen una unidad diferenciada e independiente, tanto en función de contenidos, como por la identidad de su organización responsable (1).

Dentro de una web se pueden distinguir tres bloques interrelacionados (2):

- los datos que se quiere transmitir;

- el diseño gráfico y textual; $y$

- la estructura física de los enlaces.

El presente trabajo se centra exclusivamente en los recursos seleccionados, sin pretender evaluar valores relacionados con el diseño y la estructura de cada sede web, como su navegabilidad, su ordenación, la existencia de índices temáticos, la posibilidad de vuelta a la página principal desde cualquier página, los niveles de jerarquía, la existencia o no de comentarios explicativos de los enlaces, el tamaño de las páginas, el número de accesos a la página, la uniformidad de las páginas, la presentación del contenido en la pantalla, el abuso de imágenes, etc. Este aspecto concreto ha sido estudiado por Arconada (3) que publicó un buen resumen de los criterios básicos de gestión de la información, diseño editorial, diseño gráfico y diseño de interfaz que afec$\tan$ a las web.

Tampoco se tienen en cuenta en este trabajo los recursos humanos y económicos de que dispone cada una de las sedes web a evaluar, lo que supondría necesariamente el envío de encuestas a cada una de las facultades y por alejarse también de los objetivos del trabajo. Al respecto, Merlo y Sorli (4) obtuvieron como resultado de una encuesta que el $43.63 \%$ de las sedes Web de bibliotecas eran realizadas y administradas por personal bibliotecario y que el $30 \%$ de las bibliotecas universitarias tenían páginas realizadas por informáticos.

En España existen 15 universidades en las que se imparten estudios para la obtención de la Diplomatura o la Licenciatura en Biblioteconomía y Documentación (figura 1). La Universidad Carlos III de Madrid, CEU San Pablo y la Universidad de Vic solo disponen de información docente sobre la diplomatura de Biblioteconomía. La Universidad de La Coruña no dispone todavía de sede web. Las 6 sedes web evaluadas pertenecen a las facultades de Biblioteconomía y Documentación de las siguientes Universidades:

- Universidad de Barcelona

- Universidad de León

- Universidad Complutense de Madrid

- Universidad de Murcia

- Universidad de Salamanca

- Universidad Politécnica de Valencia

Este estudio se ha realizado entre mediados de diciembre de 1998 y mediados de febrero de 1999. En el plazo de planificación y realización de trabajo, las sedes co- 
Figura 1

Url de las webs de Biblioteconomía y Documentación

\begin{tabular}{|ll|}
\hline $\begin{array}{l}\text { Universidades } \\
\text { Universidad de Barcelona. Escola Universitaria de }\end{array}$ & hrl \\
Biblioteconomía i Documentació & http://www.unileon.es/dp/dph/recursos/bibdoc \\
\hline $\begin{array}{l}\text { Universidad de León. Area de Biblioteconomía } \\
\text { y Documentación }\end{array}$ & http://www.eubd.ucm.es \\
\hline $\begin{array}{l}\text { Universidad Complutense de Madrid. Escuela Universitaria } \\
\text { de Biblioteconomía y Documentación }\end{array}$ & http://exlibris.usal.es/INDEX.HTM \\
\hline $\begin{array}{l}\text { Universidad de Salamanca. Facultad de Traducción } \\
\text { y Documentación }\end{array}$ & http://piramide.unizar.es \\
\hline $\begin{array}{l}\text { Universidad de Zaragoza. Diplomatura de Biblioteconomía } \\
\text { y Documentación. Facultad de Filosofía y Letras }\end{array}$ & Htt://alcazaba.unex.es \\
\hline $\begin{array}{l}\text { Universidad de Extremadura. Facultad de Biblioteconomía } \\
\text { y Documentación }\end{array}$ & http://fbds02.ugr.es/fbd \\
\hline Universidad de Granada. Biblioteconomía y Documentación & http://www.um.es/ reubd/ \\
\hline $\begin{array}{l}\text { Universidad de Murcia. Escuela Universitaria } \\
\text { de Biblioteconomía y Documentación }\end{array}$ & http://www.ceu.es/usp/pln/bidoc.htm \\
\hline $\begin{array}{l}\text { Universidad San Pablo CEU (Madrid). Diplomatura en } \\
\text { Biblioteconomía y Documentación }\end{array}$ & http://www.cc.alcala.es/fbd.htm \\
\hline $\begin{array}{l}\text { Universidad de Alcalá de Henares. Licenciatura } \\
\text { en Documentación }\end{array}$ & http://www.uc3m.es/dpto/BD/dpbd.htm \\
\hline $\begin{array}{l}\text { Universidad Carlos III de Madrid. Diplomatura } \\
\text { en Biblioteconomía y Documentación }\end{array}$ & http://www.uv.es/ hagonzal \\
\hline $\begin{array}{l}\text { Universidad de Valencia. Diplomatura en Biblioteconomía } \\
\text { y Documentación }\end{array}$ & $\begin{array}{l}\text { Universidad Politécnica de Valencia. Licenciatura } \\
\text { en Biblioteconomía y Documentación }\end{array}$ \\
\hline $\begin{array}{l}\text { Universidad de Vic. Diplomatura en Biblioteconomia } \\
\text { y Documentación }\end{array}$ & \\
\hline
\end{tabular}

rrespondientes a las universidades de Zaragoza y Valencia se hallaban inhabilitadas por procesos de modificación. Los recursos de la Universidad de Granada presentaban y presentan una arquitectura excesivamente distribuída, con profusión de páginas personales y enlaces ciegos («blind links») y la Universidad de Alcalá no disponía aún de páginas individualizadas dedicadas a la Biblioteconomía y Documentación.

Para poder evaluar ordenadamente los contenidos de las webs se partió de una lista de recursos de interés. Para la elaboración de esta lista de referencia de tipos de enlaces se ha tenido en cuenta la selección de recursos de Merlo y Sorli (5) así como el examen de las propias webs. Todos los recursos obtenidos se agruparon por tipos, en cuatro grandes grupos: Instituciones, Bases de Datos, Publicaciones electrónicas y Servicios. De estos grupos se efectuó el análisis de los siguientes tipos de recursos:

- Asociaciones Profesionales

- Bibliotecas Nacionales

- Bibliotecas Universitarias

- Facultades de Biblioteconomía y Documentación

- Revistas electrónicas de Biblioteconomía y Documentación 
- Conferencias y Congresos de Documentación

- Bases de datos de Documentación

- Otras bases de datos

Además, el recuento se ha limitado exclusivamente a los enlaces españoles directos. Los enlaces directos permiten la conexión online inmediata con un recurso distribuido.

Los enlaces indirectos dirigen a otras sedes a partir de las cuales se puede acceder a un recurso determinado.

Se han tabulado los enlaces directos de cada grupo de recursos de las 6 sedes Webs de Biblioteconomía y Documentación, asignando valores 061 según posean o no enlace con un recurso determinado. Se han obtenido indicadores de aporte y de aporte específico (6). Y se ha obtenido el denominador de cobertura teórica para cada grupo de recursos y globalmente, definido como la suma de todos los enlaces obtenidos por cada grupo de recursos, eliminando las repeticiones.

Se ha obtenido el índice de aporte global por sede - porcentaje de la suma total de recursos por sede sobre el denominador de cobertura teórica global. El aporte global refleja la proporción de enlaces ofrecidos por cada sede sin diferenciar ningún tipo de recurso.

Se obtiene el índice de aporte de cada sede mediante el porcentaje de enlaces proporcionados por cada sede sobre el denominador de cobertura teórica, por cada uno de los recursos. El aporte refleja la proporción de enlaces que ofrece cada sede de cada grupo de recursos.

Se obtiene el índice de aporte específico - porcentaje del número de enlaces proporcionados exclusivamente por una sola sede - a partir de los casilleros 061 de la tabla y del denominador de cobertura teórico por cada recurso y sede. El aporte específico refleja lo que cada sede ofrece específicamente y las otras sedes no ofrecen, es decir, los recursos no solapados.

Se ha obtenido la visibilidad (7) de cada recurso en las webs evaluadas. Este cálculo se ha obtenido mediante la suma de enlaces recibidos por cada recurso de cada grupo, dividido por el número de sedes y multiplicándolo por 100 .

\section{Resultados}

La lista final de recursos está compuesta por 132 enlaces directos, que constituyen el denominador de cobertura teórico global.

Para obtener el aporte de cada recurso por sede, se agrupó por códigos, obteniéndose el denominador de cobertura teórico de cada grupo de recursos: Asociaciones Profesionales 7; Bibliotecas Nacionales 5; Bibliotecas Universitarias 56; Facultades de Biblioteconomía 14; Revistas Electrónicas de Documentación 16; Conferencias y Congresos 2; Bases de datos de Documentación 5 y Otras Bases de Datos 26.

\subsection{Aporte de cada una de las sedes}

El aporte global por sede y el aporte por grupos de recursos enlazado se expresan para cada sede en la tabla 1 . 
Tabla 1

Aporte global y aporte por recursos

\begin{tabular}{|l|c|c|c|c|c|c|}
\hline & Barcelona & León & Complutense & Murcia & Salamanca & Valencia \\
\hline Global & 23,48 & 62,87 & 12,87 & 28,03 & 33,33 & 37,87 \\
Asociaciones & 85,71 & 57,14 & 42,85 & 85,71 & 28,57 & 71,42 \\
Bibliotecas nacionales & 0 & 100 & 0 & 20 & 20 & 20 \\
Bibliotecas universitarias & 7,14 & 46,42 & 3,57 & 1,78 & 5,35 & 55,35 \\
Facultades & 85,71 & 64,28 & 0 & 78,57 & 50 & 7,14 \\
Revistas electrónicas & 31,25 & 31,25 & 43,75 & 62,5 & 25 & 50 \\
Congresos & 100 & 0 & 0 & 0 & 0 & 0 \\
Bases de datos & 40 & 80 & 60 & 60 & 60 & 40 \\
Bases de datos generales & 0 & 7,69 & 7,69 & 19,23 & 92,30 & 7,69 \\
\hline
\end{tabular}

El índice de aporte específico por recursos y sedes se expresa, a continuación, en la tabla 2:

Tabla 2

Aporte especifico

\begin{tabular}{|l|c|c|c|c|c|c|}
\hline & Barcelona & Leon & Complutense & Murcia & Salamanca & Valencia \\
\hline Asociaciones & 0 & 0 & 0 & 0 & 0 & 0 \\
Bibliotecas nacionales & 0 & 80 & 0 & 0 & 0 & 0 \\
Bibliotecas universitarias & 0 & 35,71 & 0 & 0 & 3,57 & 0 \\
Facultades & 8,33 & 0 & 0 & 0 & 0 & 0 \\
Revistas electrónicas & 0 & 0 & 12,5 & 12,5 & 0 & 6,25 \\
Congresos & 100 & 0 & 0 & 0 & 0 & 0 \\
Bases de datos & 0 & 20 & 0 & 0 & 0 & 0 \\
Bases de datos generales & 0 & 0 & 0 & 3,84 & 80,76 & 0 \\
\hline
\end{tabular}

\subsection{Visibilidad de los recursos}

El número de enlaces recibidos por una sede de Internet se ha interpretado como un indicador de su visibilidad, homólogo al número de citas que reciben los autores científicos y técnicos. Estimar la visibilidad de una sede Web, sin embargo, no puede consistir en un mero recuento de enlaces a esa sede cuando se parte de un entorno determinado. En el caso del presente trabajo, el entorno está definido por las 6 colecciones de recursos examinadas y es posible ponderar la visibilidad mediante el cálculo del porcentaje de sedes que enlazan con cada uno de los recursos.

Por otra parte, las sedes Web enlazadas o citadas son heterógeneas y representan 8 grupos diferentes de recursos.

Por estas razones, se ha decidido efectuar el cálculo de la visibilidad empleando la expresión siguiente:

$\frac{\text { Número de enlaces recibidos por cada recurso de cada grupo }}{\text { Total del número de sedes }} \times 100$ 
Los resultados indican que la mayor visibilidad en cuanto al grupo de asociaciones profesionales corresponde a la Asociación Andaluza de Documentalistas (100\%). En cuanto a las bibliotecas, la Biblioteca Nacional de España entre las nacionales y la de la Universidad Complutense entre las universitarias gozan de la mayor visibilidad (66,66 en ambos casos). El centro educativo de mayor visibilidad es la Escuela de la Universidad Complutense $(\mathbf{8 3 , 3 3 \% )}$ y las revistas más visibles son Métodos de Información y Correo Bibliotecario, con idéntico porcentaje. Datatheke e ISBN son las bases de datos más enlazadas, ambas en el $83,33 \%$ de los casos. Unicamente uno de los 132 recursos alcanza una visibilidad del $100 \%$.

\section{Conclusiones}

$\mathrm{El}$ análisis del aporte global muestra la primacía de León sobre las demás sedes. Los enlaces que proporciona León superan a la Politécnica de Valencia en un 25\%; a Salamanca en un 29,54\%; a Murcia $34,84 \%$; a Barcelona $39,39 \%$ y a la Complutense de Madrid en un $50 \%$.

El aporte en Asociaciones Profesionales deja en primer lugar a Barcelona y Murcia con 85,71 , seguido por Valencia con una diferencia de $14,29 \%$; la diferencia respecto a León es de 28,57; la diferencia respecto a la Complutense de Madrid es de $42,86 \%$; por último Salamanca 57,14 .

El aporte en Bibliotecas Nacionales deja en primer lugar con un $100 \%$ a León seguida de Murcia, Salamanca, y la Politécnica de Valencia a una diferencia del $80 \%$. Barcelona y Madrid no tienen ningún enlace a este recurso.

El aporte en Bibliotecas Universitarias sitúa a León en primer lugar seguida de la Politécnica de Valencia con una diferencia de $41,17 \%$; respecto a la de Barcelona la diferencia es de $89,38 \%$; con respecto a Salamanca $91,07 \%$; con respecto a Madrid 92,85\%; con respecto a Murcia $94,64 \%$.

El aporte en Facultades de Biblioteconomía y Documentación sitúa a Barcelona seguida de Murcia con una diferencia de $7,14 \%$; luego León con una diferencia de 21,43\%; Salamanca con una diferencia de $35,71 \%$; la Politécnica de Valencia 78,57\%; y la Complutense de Madrid no tiene ningún aporte en este recurso.

El aporte en Revistas electrónicas de Documentación sitúa a Murcia en primer lugar seguida de la Politécnica de Valencia con una diferencia del 11,5\%; luego Madrid con una diferencia de $18,75 \%$; Barcelona y León con una diferencia de $30,75 \%$; y Salamanca con un $37,5 \%$ de diferencia.

El aporte en Congresos y Conferencias de Documentación sitúa a Barcelona la única con un aporte del $100 \%$.

El aporte en Bases de Datos de Documentación sitúa a León en primer lugar con un $80 \%$ y luego Madrid, Murcia y Salamanca con un $20 \%$ de diferencia y Barcelona y Politécnica con un $40 \%$ de diferencia.

El aporte en Otras Bases de Datos sitúa a Salamanca en primer lugar con un 92,3\%, seguida de Murcia con un 70,07\% de diferencia y León, Madrid y la Politécnica de Valencia a una diferencia del $84,61 \%$ y Barcelona no tienen enlaces a este tipo de recurso. sedes.

El índice de aporte específico en Asociaciones Profesionales es cero en todas las 
El índice de aporte específico en Bibliotecas Nacionales sólo existe en Barcelona con un $80 \%$,

En el caso de las Bibliotecas Universitarias existe un aporte específico en León del 35.71. seguidate Salamanca con una diferencia del $32,14 \%$ y el resto de sedes no tienen aporte especifico.

Barcelona es la única sede con aporte específico en Facultades de Biblioteconomía con un $8,33 \%$.

A Madrid y Murcia con un $12,5 \%$ de aporte específico en Revistas electrónicas de Documentación le sigue la Politécnica de Valencia con una diferencia del 6,25\%; el resto no tienen aporte específico en este grupo de recursos.

Barcelona es la única sede con aporte específico en Congresos y Conferencias de Documentación con un $100 \%$.

León es la única sede con índice de aporte específico en Bases de Datos de Documentación con un porcentaje del $20 \%$.

El índice de aporte específico en Otras Bases de Datos sitúa a Salamanca con un $80,76 \%$ seguida de Murcia con una diferencia del $76,92 \%$. El resto no poseen aporte específico en este grupo de recursos.

Durante el transcurso del trabajo se han podido constatar diversas situaciones que afectan al contenido de este trabajo, destacando entre las más significativas:

- La actualización constante de los contenidos de las sedes con adiciones de información implica una obsolescencia de la evaluación en un corto periodo de tiempo.

- Las revisiones completas de webs dejan temporalmente fuera de servicio algunas sedes, como ocurrió en el caso del servidor Pirámide de la Universidad de Zaragoza que no se pudo evaluar por este motivo.

- Los cambios frecuentes en las direcciones no sólo de las sedes a estudiar sino. también de sus enlaces, suponen una considerable pérdida de tiempo y, por otro lado, dejan inaccesibles enlaces que en otros momentos eran válidos. Consideramos una buena recomendación dirigida a los encargados de la realización de cada página que mantengan la vieja dirección con el aviso del cambio y la nueva dirección para evitar dichos problemas a un menor coste.

- La denominación de los recursos ha sido otra de las dificultades para la elaboración del trabajo, no sólo por no coincidir en ocasiones con la denominación de nuestros grupos de recursos, sino por la dificultad de encontrar determinados enlaces bajo títulos poco indicativos de contener determinada información, por ejemplo en la web de Salamanca las bases de datos estaban bajo un enlace llamado «Motores de búsqueda.»

\section{Agradecimientos}

El presente estudio constiłuyó el trabajo de investigación de la asignatura Investigación Evaluativa en Documentación, dirigido por la profesora María Francisca Abad García, dentro del programa de tercer ciclo del Departamento de Historia de la Ciencia y Documentación de la Universidad de Valencia. La sugerencia de su realización y el enfoque metodológico empleado se deben a la Dra. Abad. 


\section{Referencias bibliograficas}

1. AGUILLO, I. F. Hacia un concepto documental de sede Web. Information World en Espanol. 1998, n. ${ }^{\circ} 1-2$, p. $45-46$

2. TERMENS I GRAELLS, M. Les webs de les biblioteques de Catalunya: estructura interna i enllaços. En: 6es. Jornades Catalanes de Documentacio, Barcelona, 23 a 25 d'Octubre, 1997 , p. $507-517$

3. ARCONADA, P. Sitios Web con estilo. Iword 1997, n. $^{\circ} 10$, p. 26-36

4. MERLO VEGA, J. A.; SORLI ROJO, A. Las bibliotecas como clientes y servidoras de información Web. En: 6es. Jormades Catalanes de Documentació, Barcelona, 23 a 25 d'Octubre, 1997, p. $317-327$

5. MERLO VEGA, J. ; SORLI ROJO, A. Biblioteconomía y Documentación en Internet. Madrid: CINDOC, 1997

6. ABAD GARCIA, M.; ALEIXANDRE BENAVENT, R.; PERIS BONET, R. Estrategias de búsqueda de artículos de revistas españolas. Estudio de un caso: evaluación de la calidad de los sistemas de información. Gaceta Sanitaria, 1995, n. ${ }^{\circ}$ 51, p. 363-370

7. RODRIGUEZ GAIRIN, J. Valoración del impacto de la información en Internet: Altavista, el «Citation Index» de la Red. Revista Española de Documentación Científica, 1997, vol. 20, n. $^{\circ} 2$, p. $175-181$ 